\title{
Efficacy and safety of PDI/PDLI blockades versus docetaxel in patients with pretreated advanced non-small-cell lung cancer: a meta-analysis
}

This article was published in the following Dove Press journal:

OncoTargets and Therapy

\author{
Jixiang Liu \\ Yulan Zhong \\ Shanshan Peng \\ Xiangxiang Zhou \\ Xin Gan
}

Department of Respiratory Medicine, The First Affiliated Hospital, Nanchang University, Nanchang, China
Correspondence: Xin Gan

Department of Respiratory Medicine,

The First Affiliated Hospital, Nanchang

University, 17 Yongwaizheng Street.

Nanchang, Jiangxi 330006, China

Email ganxin 1207@I26.com
Background: PD1/PDL1 blockade is a promising treatment for patients with non-small-cell lung cancer (NSCLC). Here, we employed meta-analysis to evaluate the efficacy and safety of PD1/PDL1 blockades for previously treated NSCLC patients.

Methods: Randomized clinical trials were retrieved by searching electronic databases. Data for HRs, 95\% CIs for overall survival (OS), progression-free survival (PFS), and adverse events (AEs) were extracted and pooled.

Results: A total of five randomized controlled trials including 2,910 patients were included in this meta-analysis. Pooled HRs $(95 \% \mathrm{CI})$ were $0.71(0.63-0.79, P<0.0001)$ for OS and $0.86(0.73-1.02)$ for PFS. In the subgroup analysis, the pooled HR (95\% CI) for PFS was $0.82(0.75-0.91, P<0.0001)$ in patients with high PDL1 expression, but no significant difference was seen in patients with low expression ( 0.97 [0.76-1.24], $P=0.82)$. The pooled RR for treatment-related AEs of all grades was $0.32(0.27-0.38, P<0.00001)$ compared with the docetaxel arm, while that for grade 3-5 treatment-related AEs in the PD1/PDL1-blockade arm was $0.16(0.10-0.27, P<0.00001)$.

Conclusion: PD1/PDL1 blockades enhanced OS and PFS and led to lower risk of AEs in NSCLC patients. Smoking history and wild-type EGFR were associated with extended OS.

Keywords: non-small-cell lung cancer, checkpoint immunologic treatments, PD1, PDL1, meta-analysis

\section{Introduction}

In recent decades, lung cancer has been one of the most commonly diagnosed malignancies, and it is the leading cause of cancer-related deaths worldwide. ${ }^{1}$ Lung cancer-survival rates gradually fall beyond 5 years after diagnosis. ${ }^{2,3}$ Non-small-cell lung cancer (NSCLC) patients account for $\sim 85 \%$ of all cases of lung cancer. Most are diagnosed with locally advanced or metastatic disease, and 5-year survival rates remain at $<17 \%{ }^{4}$

Platinum-based chemotherapy yields a superior quality of life compared to best supportive care for advanced NSCLC patients during or after standard chemotherapy; however, in most patients, the disease will progress. ${ }^{5-7}$ Docetaxel has been selected as the standard second-line chemotherapeutic agent, based on the results of clinical trials. ${ }^{8,9}$ It can achieve an improved overall response rate (ORR) in advanced NSCLC patients treated with first-line chemotherapy; however, the disease still progresses rapidly during or after monochemotherapy, including docetaxel therapy; hence, outcomes are still unsatisfactory. In recent decades, targeted therapies, such as EGFR and ALK-tyrosine kinases, have been of great value to patients for whom conventional chemotherapy is 
not suitable. However, their applications are limited by the high risk of drug resistance, which commonly occurs. ${ }^{10-12}$

PD1 and PDL1 blockades have been used as immunocheckpoint therapies, and have shown promising results in clinical trials in patients with epithelial cancers, such as NSCLC, melanoma, and renal-cell carcinoma. ${ }^{13}$ The PD1 receptor is expressed by activated T and B cells. The combination of PD1 and PDL1 suppresses T cells through a feedback loop, leading to tumor immunoevasion. The mechanism underlying this effect may be that tumor cells escape recognition and elimination by the immune system. ${ }^{14,15}$ Early-phase clinical trials have shown that these drugs can produce durable antitumor responses in advanced NSCLC. ${ }^{16-18}$ However, the benefits for patients treated with these blockades are still unclear, as most of the relevant trials showed no significant improvement in survival. It is also uncertain which groups of patients may benefit from immunocheckpoint therapy. In view of the limited sample sizes of the clinical trials, it is impossible to draw a conclusion based on any given study. Therefore, this study aimed to synthetically evaluate the efficacy and safety of PD1/PDL1 blockades vs docetaxel in patients with pretreated advanced NSCLC. Subgroup analysis was also performed to determine which patients are more likely to benefit.

\section{Methods}

\section{Search strategy}

In order to retrieve all relevant studies published in English, a rigorous search for reports of clinical trials from PubMed, Embase, and the Cochrane Library was undertaken. The most recent report was dated December 27, 2017. Conference abstracts from the American Society of Clinical Oncology, European Society for Medical Oncology, and International Association for the Study of Lung Cancer were also evaluated. Keywords used for the search were as follows: (nonsmall-cell lung cancer OR NSCLC) AND (pembrolizumab OR Keytruda OR MK-3475 OR lambrolizumab OR nivolumab OR BMS-936558 OR Opdivo OR MDX-1106 OR atezolizumab OR MPDL3280A OR Tecentrip). There was no restriction by year.

\section{Outcome for analysis}

Outcomes analyzed for efficacy were overall survival (OS), progression-free survival (PFS), and ORR. Outcomes analyzed for safety were treatment-related adverse events (TRAEs), including any grade and grades 3-5.

\section{Selection and exclusion criteria}

All relevant articles underwent evaluation for eligibility by two investigators independently, and articles were selected according to criteria of the study being a randomized controlled trial (RCT), a comparison with docetaxel having been performed, at least one efficacy outcome and one safety outcome reported, and the full text being available. We excluded letters, expert opinions, case reports, reviews, articles without available data, and duplicate publications.

\section{Quality assessment}

We assessed the quality of included clinical RCTs according to the criteria presented in the Cochrane Handbook for Systematic Reviews of Interventions (version 5.1.0, chapter 8), and evaluated random-sequence generation, allocation concealment, blinding of participants and personnel, blinding of outcome assessment, and incomplete outcome data. To ensure a low incidence of bias among the studies included, such issues as selective bias were also considered. ${ }^{19}$

\section{Data extraction}

The first author, date of publication, interventions for experimental and control groups, number of patients enrolled in each trial, patient details (age, sex, expression level of PDL1, pathological type, smoking history, and EGFR status), ORR, HRs with 95\% CIs and $P$-values for OS and PFS and for any-grade and grade 3-5 TRAEs were extracted by two individual investigators independently. We also collected information by subgroup to obtain sufficient data for our subgroup analyses.

\section{Statistical analysis}

Pooled HRs with 95\% CIs for OS and PFS and ORs for dichotomous data (ORR and TRAEs) with 95\% CIs were calculated using RevMan 5.3. HRs $<1$ favored the PD1/ PDL1-blockade arm, whereas HRs $>1$ favored the docetaxel arm. ORs $>1$ for ORR and TRAEs reflected higher overall response and toxicity, respectively, in the immunotherapy arm. $P<0.05$ was considered significant. Heterogeneity across studies was assessed by a combination of the standard $Q$ test and $I^{2}$ test: results with $P<0.10$ or $I^{2}>50 \%$ were considered statistically significant. A fixed-effect model was employed when heterogeneity was absent. Otherwise, a random-effect model was appropriate. Subgroup analysis was conducted according to expression level of PDL1, pathological type, smoking history, and EGFR status.

\section{Publication bias}

An extensive search strategy was used to minimize the potential for publication bias. Graphic funnel plots were generated to assess publication bias visually. If publication bias was 
found visually, the Begg-Mazumdar rank-correlation test and Egger regression-asymmetry test were used. ${ }^{20,21}$

\section{Results}

\section{Study identification}

Using the outlined search strategy, a total of 1,990 records were obtained, from which 1,240 duplicates were removed. After screening, 742 articles, including reviews, case reports, and non-RCTs, were excluded. Of the remaining 8 records, three studies did not report relevant data. For the remaining five studies, the two reviewers were in perfect agreement with respect to eligibility and assessed the quality of the studies independently by the scoring criteria in the Cochrane handbook. The study selection process is presented in Figure 1. The risk-of-bias graph and summary of selected studies generated by RevMan 5.3 are shown in Figure 2 .

\section{Characteristics of studies}

We enrolled 2,915 patients with advanced NSCLC in five RCTs. Experimental interventions used in those trials were PD1/PDL1 blockades, including nivolumab, pembrolizumab, and atezolizumab, with docetaxel treatment as a control. One of the trials was phase 2 , one was phase $2 / 3$, and three were phase 3 . We collected the basic characteristics of patients in each included trial and extracted information to obtain relevant information for our analysis. We defined the cutoff for high PDL1 expression as 1\%, ie, membranous PDL1 staining in at least $1 \%$ of tumor cells. Details of included studies, authors, numbers of patients, interventions, basic characteristics of patients, and ORRs and HRs for OS and PFS, are summarized in Table 1.

\section{Meta-analyses}

Pooled HR values showed significant improvement in OS for PD1/PDL1 blockades (HR 0.71, 95\% CI 0.63-0.79; $P<0.00001$ ), but not in PFS (HR 0.86, 95\% CI 0.73-1.02; $P=0.09$; Figure 3). Pooled ORs for ORR also indicated a benefit of PD1/PDL1 blockades (OR 1.64, 95\% CI 1.19-2.26; $P=0.003$ ). Subgroup analysis according to PDL1 expression of tumors showed that immunotherapy significantly improved PFS in patients with high PDL1 expression (HR $0.82,95 \%$ CI $0.75-0.91 ; P<0.0001)$, but not in those with

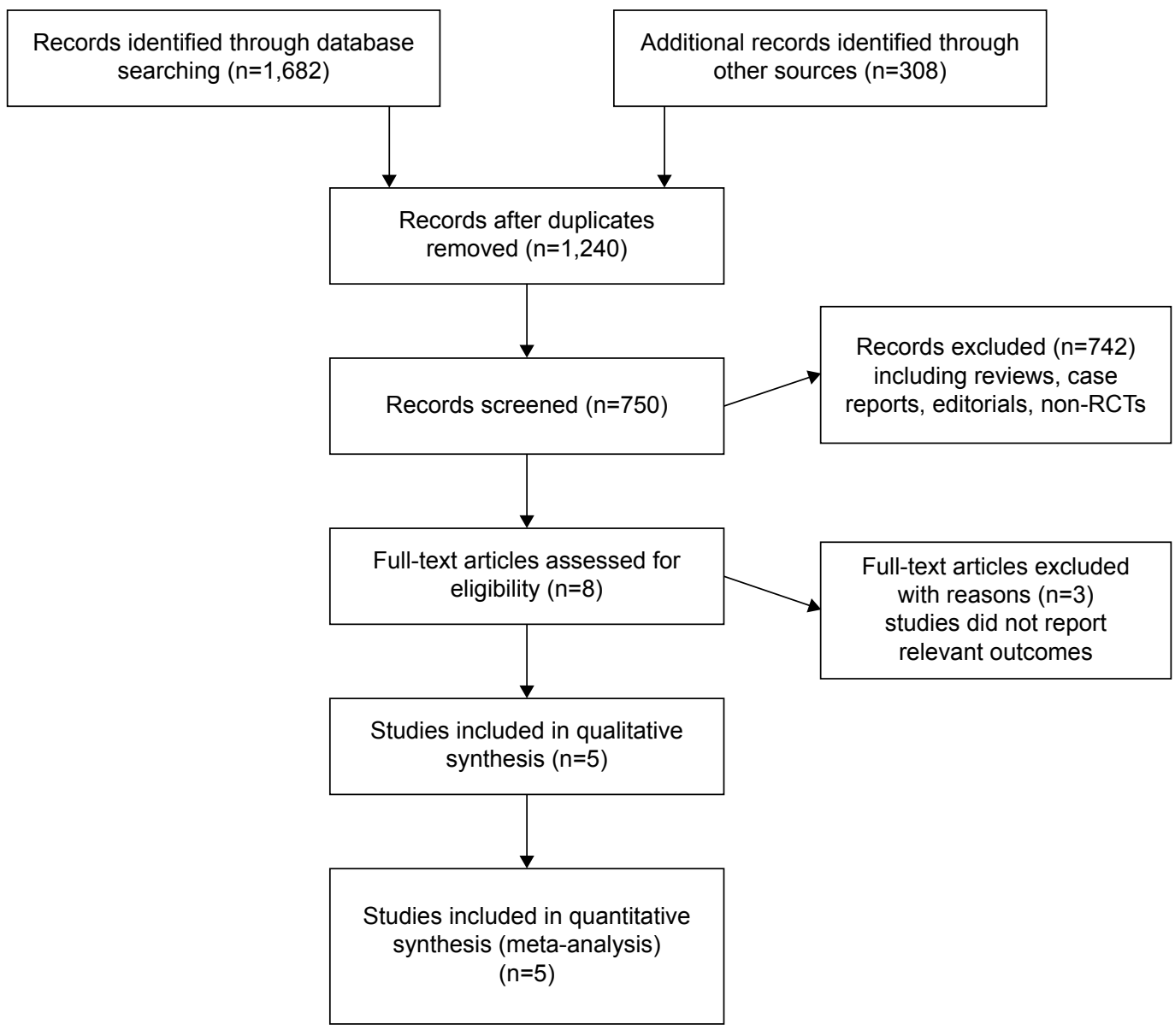

Figure I Flowchart of study selection procedure. 


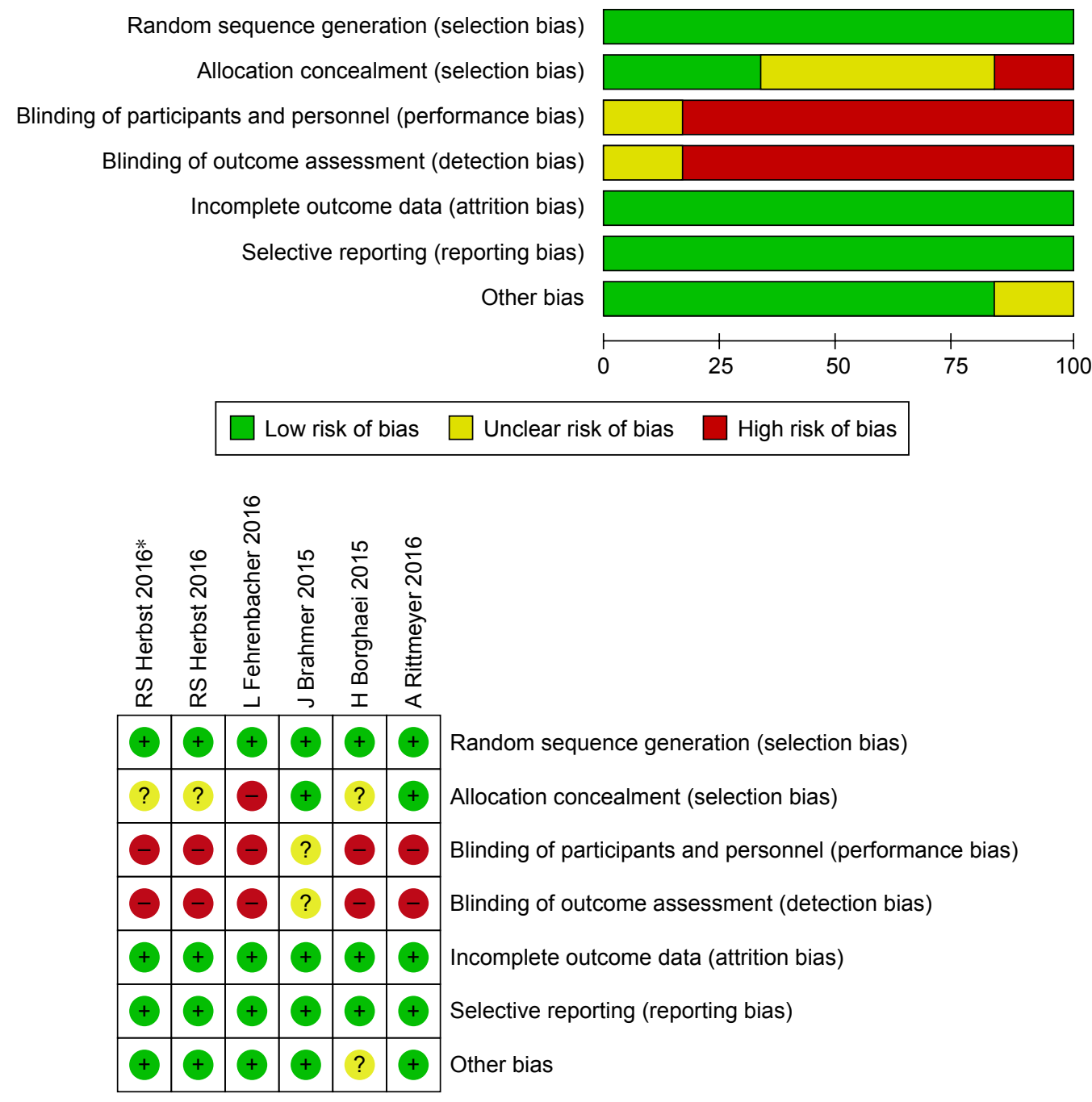

Figure 2 Risk of bias and summary of included clinical trials.

low expression (HR 0.97, 95\% CI 0.76-1.24; $P=0.82$ ). There was no significant difference in OS between patients with high (HR 0.62) and low (HR 0.79) expression. Other subgroup analyses of OS according to pathological type, smoking history, and EGFR status all showed improvement in OS associated with PD1/PDL1 blockade (Figure 4).

In the safety analysis, meta-analysis showed that PD1/PDL1 blockades were associated with a significant decrease in any-grade TRAEs (RR $0.32,95 \%$ CI $0.27-0.38$; $P<0.00001)$ compared with the docetaxel arm. The pooled RR of grade 3-5 TRAEs in the PD1/PDL1 blockade arm was $0.16(95 \%$ CI $0.10-0.27, P<0.00001)$ compared with the docetaxel arm (Figure 5).

\section{Sensitivity analyses}

Sensitivity analyses were conducted by excluding studies one by one. The results showed no significant differences when compared with the former summary estimates and had excellent stability.

\section{Publication bias}

No significant publication bias was observed for any outcome, as determined by funnel plots constructed with RevMan 5.3.

\section{Discussion}

After or during first-line chemotherapy, some patients still have a risk of disease progression, defined as development of new lesions or detection of $>20 \%$ increase in the size of the primary lesion. ${ }^{22}$ Previous studies have found that some tumor cells can evade immunological recognition and destruction by expressing PDL1, suggesting a potentially effective antitumor strategy. Monoclonal antibodies targeting PD1/PDL1, including those used in standard second-line 


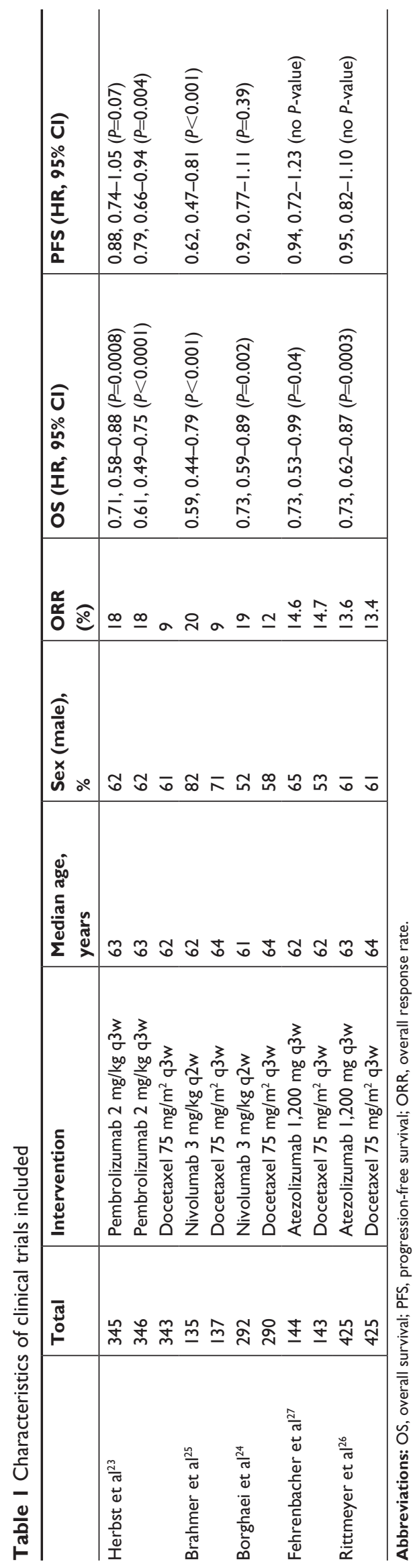

therapy for NSCLC, such as nivolumab, pembrolizumab, and atezolizumab, can restore the tumor-suppression effect of T cells by blocking the PDL1 signaling pathway. Many phase 1 trials of immunotherapy have shown promising improvements in OS and PFS, as well as lower incidence of TRAEs with an appropriate dose. ${ }^{16-18}$ Furthermore, several trials compared PD1/PDL1 blockades with docetaxel with respect to efficacy and safety. ${ }^{23-27}$ To further validate PD1/ PDL1 blockade monotherapies, the efficacy and safety of PD1/PDL1 blockades in pretreated advanced NSCLC patients were systematically analyzed in this work.

In this meta-analysis, we evaluated the efficacy of PD1/PDL1 agents compared with docetaxel in pretreated advanced NSCLC patients, selecting OS and PFS as the primary outcomes and ORR as the secondary outcome. The results showed that OS improved with PD1/PDL1 blockades (median 13.8 months) vs docetaxel (median 9.6 months) on an intention-to-treat basis. ${ }^{27}$ The pooled HR and its $P$-value indicated that patients receiving immunotherapy had better OS than those treated with docetaxel. The results were not promising for PFS, however, with $P>0.05$. In addition, the pooled OR results suggested an increase in ORR from 13.6\% to $20 \%$ between the PD1/PDL1 group and the docetaxel group. As such, it was concluded that PD1/PDL1 therapy led to significant improvements in OS and ORR, but not in PFS. High PDL1 expression levels in tumor cells may contribute to improvements in OS and PFS.

In order to test our hypothesis, subgroup analysis was performed according to PDL1 expression level of tumor cells. High expression was defined as $>1 \%$, as the data from the five clinical trials included were most abundant in the range of $1 \%-50 \%$. This low cutoff value for high expression levels enabled us to include the maximum number of patients who could benefit from the therapy. In the subgroup analysis, the pooled HR suggested a significant improvement in PFS in the immunotherapy arm compared with that in the docetaxel arm. Although the HR for OS in patients with high PDL1 expression was slightly lower than that for the whole population, PDL1 expression levels may be inadequate to predict OS benefit, in contrast with docetaxel, for which both high expression and low expression groups can gain improvement in OS from PD1/PDL1 blockades. Patient tendency to change their choice of therapy after an unsuccessful course of treatment has led to disease progression. In contrast to OS, PFS was measured before patients changed their therapeutic schedules, and thus was not affected by subsequent therapies. Therefore, PDL1 expression could be used as a biomarker to predict PFS improvement. However, 


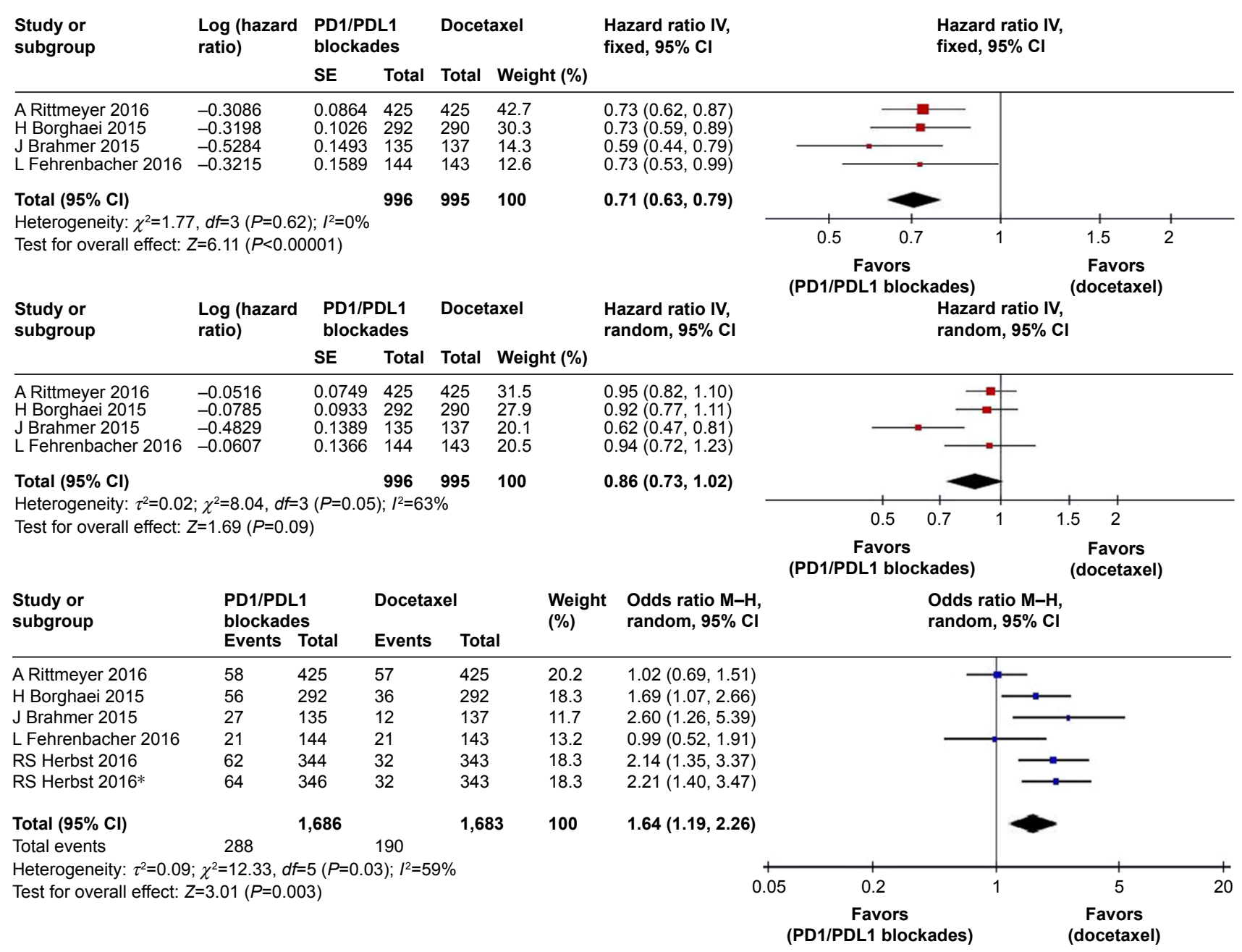

Figure 3 Forest plots for OS, PFS, and ORR for PDI/PDLI blockades vs docetaxel.

Note: *Represents data in this article that compares different doses of Pembrolizumab with docetaxel.

Abbreviations: OS, overall survival; PFS, progression-free survival; ORR, overall response rate.

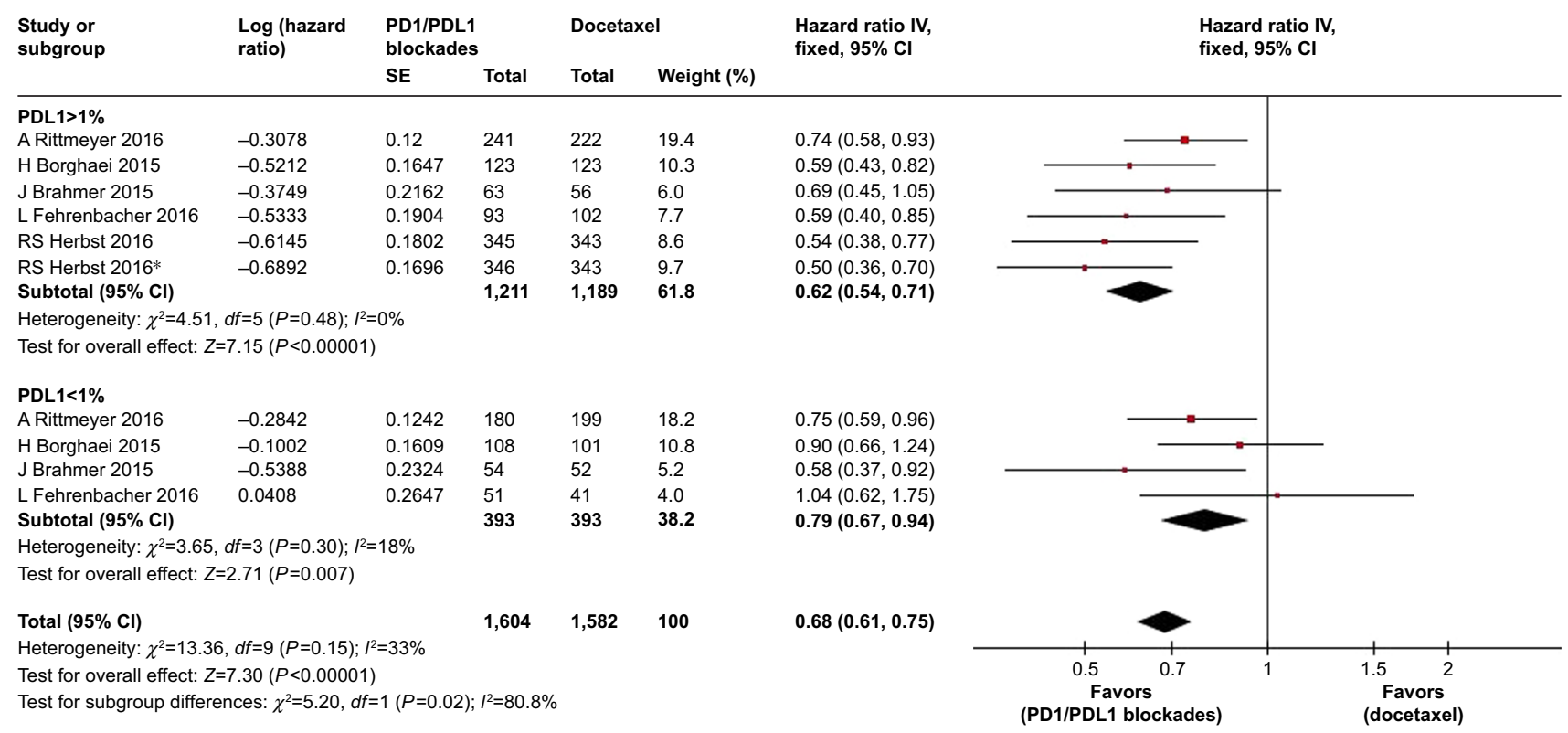

Figure 4 (Continued) 


\begin{tabular}{|c|c|c|c|c|c|}
\hline \multirow[t]{2}{*}{$\begin{array}{l}\text { Study or } \\
\text { subgroup }\end{array}$} & \multirow[t]{2}{*}{$\begin{array}{l}\text { Log (hazard } \\
\text { ratio) }\end{array}$} & \multicolumn{2}{|c|}{$\begin{array}{l}\text { PD1/PDL1 } \\
\text { blockades }\end{array}$} & \multicolumn{2}{|c|}{ Docetaxel } \\
\hline & & SE & Total & Total & Weight $(\%)$ \\
\hline \multicolumn{6}{|l|}{ PDL1>1\% } \\
\hline A Rittmeyer 2016 & -0.0939 & 0.1057 & 241 & 222 & 19.0 \\
\hline H Borghaei 2015 & -0.3512 & 0.1476 & 123 & 123 & 9.8 \\
\hline J Brahmer 2015 & -0.4105 & 0.2094 & 63 & 56 & 4.9 \\
\hline RS Herbst 2016 & -0.1262 & 0.0893 & 345 & 343 & 26.7 \\
\hline RS Herbst 2016* & -0.2387 & 0.0902 & 346 & 343 & 26.1 \\
\hline Subtotal $(95 \% \mathrm{Cl})$ & & & 1,118 & 1,087 & 86.5 \\
\hline \multicolumn{6}{|c|}{ Heterogeneity: $\chi^{2}=3.92, d f=4(P=0.42) ; I^{2}=0 \%$} \\
\hline \multicolumn{6}{|c|}{ Test for overall effect: $Z=3.92(P<0.0001)$} \\
\hline \multicolumn{6}{|l|}{ PDL1<1\% } \\
\hline H Borghaei 2015 & 0.1742 & 0.1541 & 108 & 101 & 9.0 \\
\hline J Brahmer 2015 & -0.422 & 0.2153 & 54 & 52 & 4.6 \\
\hline Subtotal $(95 \% \mathrm{Cl})$ & & & 162 & 153 & 13.5 \\
\hline \multicolumn{6}{|c|}{ Heterogeneity: $\chi^{2}=5.07, d f=1(P=0.02) ; I^{2}=80 \%$} \\
\hline \multicolumn{6}{|c|}{ Test for overall effect: $Z=0.22(P=0.82)$} \\
\hline Total $(95 \% \mathrm{Cl})$ & & & 1,280 & 1,240 & 100 \\
\hline \multicolumn{6}{|c|}{ Heterogeneity: $\chi^{2}=10.52, d f=6(P=0.10) ; l^{2}=43 \%$} \\
\hline \multicolumn{6}{|c|}{ Test for overall effect: $Z=3.73(P=0.0002)$} \\
\hline \multicolumn{6}{|c|}{ Test for subgroup differences: $\chi^{2}=1.53, d f=1(P=0.22) ; l^{2}=34.6 \%$} \\
\hline
\end{tabular}

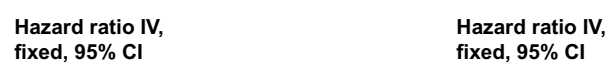

$1.19(0.88,1.61)$ $0.66(0.43,1.00)$ $0.97(0.76,1.24)$

$0.84(0.77,0.92)$

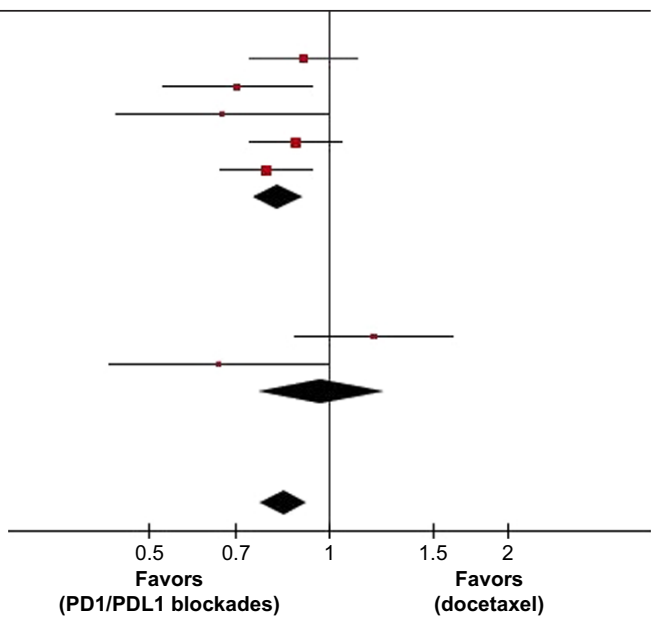

Study or Log (hazard

PD1/PDL1

Docetaxel

Hazard ratio IV, fixed, $95 \% \mathrm{Cl}$

Hazard ratio IV, fixed, $95 \% \mathrm{Cl}$

\begin{tabular}{lllllll} 
subgroup & ratio) & \multicolumn{2}{c}{ blockades } & & fixed, 95\% Cl \\
& & SE & Total & Total & Weight (\%) & \\
\hline EGFR wild & & & & & & \\
A Rittmeyer 2016 & -0.3655 & 0.0914 & 318 & 310 & 37.4 & $0.69(0.58,0.83)$ \\
H Borghaei 2015 & -0.4121 & 0.1333 & 168 & 172 & 17.6 & $0.66(0.51,0.86)$ \\
RS Herbst 2016 & -0.4105 & 0.0956 & 581 & 294 & 34.2 & $0.66(0.55,0.80)$ \\
Subtotal (95\% Cl) & & & $\mathbf{1 , 0 6 7}$ & $\mathbf{7 7 6}$ & $\mathbf{8 9 . 2}$ & $\mathbf{0 . 6 8 ( 0 . 6 0 , 0 . 7 6 )}$
\end{tabular}

Heterogeneity: $\chi^{2}=0.14, d f=2(P=0.93) ; l^{2}=0 \%$

Test for overall effect: $Z=6.62(P<0.00001)$

EGFR mutant

A Rittmeyer 2016

A Rittmeyer 2016

RS Herbst 2016

\subsection{4}

0.1615

$-0.1328$

\subsection{2}

$42 \quad 43$

$\begin{array}{ll}43 & 3.8 \\ 38 & 4.2\end{array}$

3.8
4.2

$1.24(0.71,2.18)$

$1.18(0.69,2.00)$

$0.88(0.45,1.70)$

Subtotal $(95 \% \mathrm{Cl})$

$\begin{array}{lll}60 & 26 & 2.7 \\ 146 & 107 & 10.8\end{array}$

$1.11(0.80,1.55)$

Heterogeneity: $\chi^{2}=0.69, d f=2(P=0.71) ; l^{2}=0 \%$

Test for overall effect: $Z=0.63(P=0.53)$

Total $(95 \% \mathrm{Cl})$

$1,213 \quad 883 \quad 100$

$0.71(0.64,0.80)$

Heterogeneity: $\chi^{2}=8.50, d f=5(P=0.13) ; l^{2}=41 \%$

Test for overall effect: $Z=6.05(P<0.00001)$

Test for subgroup differences: $\chi^{2}=7.66, d f=1(P=0.006) ; l^{2}=87.0 \%$

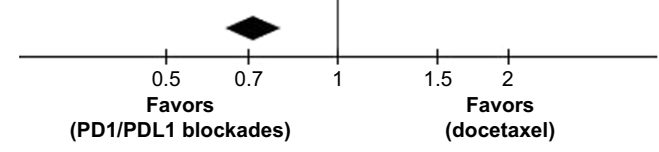

\begin{tabular}{|c|c|c|c|c|c|c|c|c|}
\hline \multirow[t]{2}{*}{$\begin{array}{l}\text { Study or } \\
\text { subgroup }\end{array}$} & \multirow[t]{2}{*}{$\begin{array}{l}\text { Log (hazard } \\
\text { ratio) }\end{array}$} & \multicolumn{2}{|c|}{$\begin{array}{l}\text { PD1/PDL1 } \\
\text { blockades }\end{array}$} & \multicolumn{2}{|c|}{ Docetaxel } & \multirow[t]{2}{*}{$\begin{array}{l}\text { Hazard ratio IV, } \\
\text { fixed, } 95 \% \mathrm{Cl}\end{array}$} & \multirow{2}{*}{\multicolumn{2}{|c|}{$\begin{array}{l}\text { Hazard ratio IV, } \\
\text { fixed, } 95 \% \mathrm{Cl}\end{array}$}} \\
\hline & & SE & Total & Total & Weight (\%) & & & \\
\hline \multicolumn{9}{|l|}{ Smoker } \\
\hline A Rittmeyer 2016 & -0.3077 & 0.0935 & 341 & 353 & 35.3 & $0.74(0.61,0.88)$ & - & \\
\hline H Borghaei 2015 & -0.3635 & 0.1085 & 231 & 227 & 26.2 & $0.70(0.56,0.86)$ & $-=$ & \\
\hline J Brahmer 2015 & -0.5221 & 0.1525 & 121 & 129 & 13.3 & $0.59(044,0.80)$ & & \\
\hline L Fehrenbacher 2016 & -0.2885 & 0.1672 & 117 & 114 & 11.0 & $0.75(0.54,1.04)$ & & \\
\hline Subtotal $(95 \% \mathrm{Cl})$ & & & 810 & 823 & 85.8 & $0.70(0.62,0.79)$ & & \\
\hline
\end{tabular}

Heterogeneity: $\chi^{2}=1.62, d f=3(P=0.65) ; l^{2}=0 \%$

Test for overall effect: $Z=5.93(P<0.00001)$

Never smoker

A Rittmeyer 2016

H Borghaei 2015

L Fehrenbacher 2016

\section{$-0.339$} 0.015

$-0.602$

0.2122

0.2353

0.421

Subtotal $(95 \%$ C $)$

Heterogeneity: $\chi^{2}=2.13, d f=2(P=0.34) ; l^{2}=6 \%$

Test for overall effect: $Z=1.57(P=0.12)$

Total $(95 \% \mathrm{Cl})$

Heterogeneity: $\chi^{2}=4.35, d f=6(P=0.63) ; l^{2}=0 \%$

Test for overall effect: $Z=6.08(P<0.00001)$

Test for subgroup differences: $\chi^{2}=0.60, d f=1(P=0.44) ; I^{2}=0 \%$

$\begin{array}{llll}84 & 72 & 6.9 & 0.71(0.47,1.08) \\ 58 & 60 & 5.6 & 1.02(0.64,1.61) \\ 27 & 29 & 1.7 & 0.55(0.24,1.25) \\ \mathbf{1 6 9} & \mathbf{1 6 1} & \mathbf{1 4 . 2} & \mathbf{0 . 7 9}(\mathbf{0 . 5 9 , 1 . 0 6})\end{array}$

$0.79(0.59,1.06)$

$0.71(0.64,0.80)$

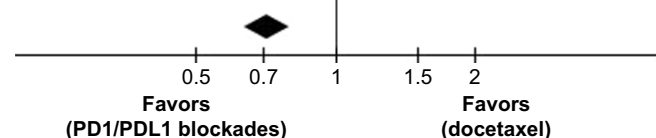

Figure 4 Forest plots for OS and PFS in patients with different PDLI expression levels, EGFR status, and smoking history.

Note: *Represents data in this article that compares different doses of Pembrolizumab with docetaxel.

Abbreviations: OS, overall survival; PFS, progression-free survival. 


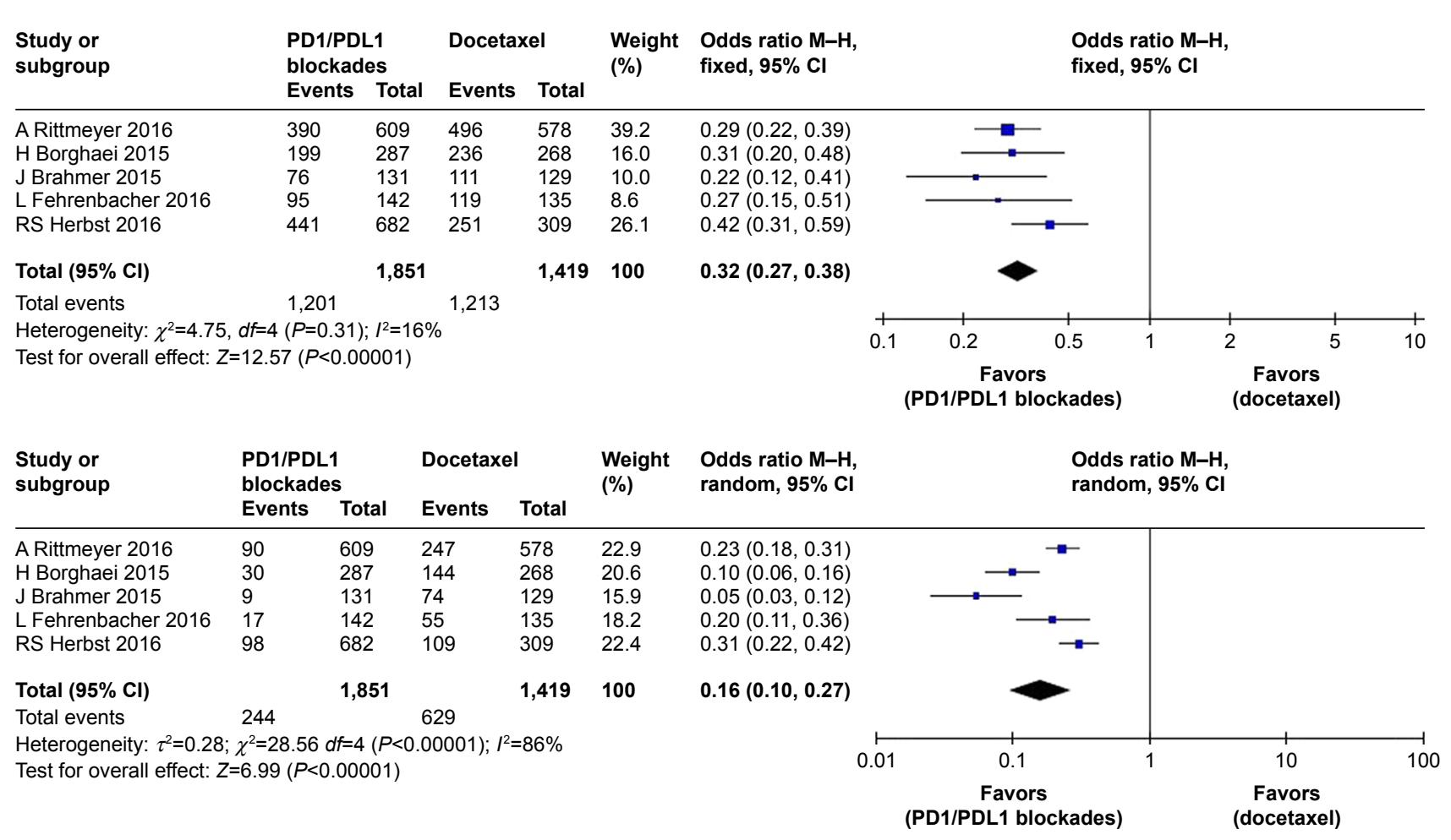

Figure 5 Forest plots for incidence of any-grade and grade 3-5 treatment-related adverse events.

PDL1 expression evaluation remains a challenge for clinicians. PDL1 expression levels of tumors are not very stable, and may be influenced by activated tumor antigen-specific T cells. ${ }^{28,29}$ The immunohistochemistry assays of PDL1 employed in the selected studies used Dako, clone 28-8 (Epitomic), and 22C3 antibody (Merck). There is no single standard method to evaluate levels of PDL1 expression. In addition, it is unclear whether peripheral blood profiling can be used to detect responses to PDL1 blockade..$^{30}$ Therefore, there is an urgent need for clinicians to find a stable way to detect PDL1 expression levels.

To benefit more patients, further subgroup analyses were performed, but only EGFR status and smoking history showed promising results. NSCLC patients with wild-type EGFR or smoking history showed improved OS in the PD1/ PDL1 group compared to that of the control group receiving docetaxel monotherapy; however, no such effect was seen for patients with EGFR mutation and a no-smoking history. Azuma et al's research showed that the presence of EGFR mutations was significantly associated with increased PDL1 expression as an independent hazard factor. ${ }^{31}$ However, EGFR mutation may also be associated with better outcomes because of molecularly targeted therapies. As such, it is unclear whether EGFR status or PDL1 expression should be considered a potential novel predictive biomarker for advanced NSCLC patients undergoing immunocheckpoint therapies. Higher expression levels of PDL1 were more frequently found in smokers and were influenced by number of pack-years. ${ }^{32,33}$ However, these studies' samples were too small for conclusions to be drawn about best practice for all NSCLC patients. Therefore, factors influencing whether NSCLS patients will benefit from PD1/PDL1 blockades are so far unknown and merit further investigation.

The safety of PD1/PDL1 blockades compared with docetaxel was evaluated in this work. Although Herbst et al's trial ${ }^{23}$ was aimed at NSCLC patients with PDL1 expression $>1 \%$, it was included because it contained a valuable evaluation of safety. Pooled ORs for any-grade and grades 3-5 TRAEs were 0.32 and 0.16 , respectively; this indicated that PD1/PDL1 agents were superior to docetaxel in terms of safety. The most common TRAEs in the original studies were fatigue, hematological adverse events (AEs), and gastrointestinal reactions. Hematological AEs, such as anemia and neutropenia, and gastrointestinal reactions, such as nausea, decreased appetite, and diarrhea, were significantly less common with anti-PD1/PDL1 therapy. ${ }^{34}$ This may have been because docetaxel has many general properties of chemotherapy and can hence damage epithelium-derived cells, while anti-PD1/PDL1 blockades do not. However as an immunocheckpoint therapy, PD1/PDL1 blockades are associated with increased incidence of immunorelated AEs, including pneumonitis and hypothyroidism, compared with 
docetaxel. Therefore, clinicians need to consider potential AEs more carefully when using PD1/PDL1 blockades.

\section{Conclusion}

Based on these findings, we can conclude that PD1/PDL1 blockades improve OS and ORR in the intention-to-treat population, but not PFS. Subgroup analysis revealed that improvement in PFS is associated with high PDL1 expression and that patients with wild-type EGFR or smoking history may see a benefit in terms of OS. PD1/PDL1 inhibitors carry a lower risk of any-grade and serious TRAEs than docetaxel, but AEs related to immunoreactions should still be monitored closely.

\section{Acknowledgment}

The cost of printing all of literatures was provided by Major Project of Jiangxi Natural Science Foundation, Grant/Award Number:20161ACB20012 and the National Natural Science Foundation of China, Grant/Award Number:81260004.

\section{Disclosure}

The authors report no conflicts of interest in this work.

\section{References}

1. Miller KD, Siegel RL, Lin CC, et al. Cancer treatment and survivorship statistics, 2016. CA Cancer J Clin. 2016;66(4):271-289.

2. Siegel RL, Miller KD, Jemal A. Cancer statistics, 2016. CA Cancer J Clin. 2016;66(1):7-30.

3. Torre LA, Siegel RL, Jemal A. Lung cancer statistics. Adv Exp Med Biol. 2016;893:1-19.

4. Howlader N, Chen VW, Ries LA, et al. Overview of breast cancer collaborative stage data items - their definitions, quality, usage, and clinical implications: a review of SEER data for 2004-2010. Cancer. 2014;120(Suppl 23):3771-3780.

5. Paramanathan A, Solomon B, Collins M, et al. Patients treated with platinum-doublet chemotherapy for advanced non-small-cell lung cancer have inferior outcomes if previously treated with platinum-based chemoradiation. Clin Lung Cancer. 2013;14(5):508-512.

6. Dahlberg SE, Schiller JH, Bonomi PB, et al. Body mass index and its association with clinical outcomes for advanced non-small-cell lung cancer patients enrolled on Eastern Cooperative Oncology Group clinical trials. J Thorac Oncol. 2013;8(9):1121-1127.

7. Ettinger DS, Wood DE, Akerley W, et al. NCCN guidelines insights: non-small cell lung cancer, Version 4.2016. J Natl Compr Canc Netw. 2016;14(3):255-264.

8. Fossella FV, Devore R, Kerr RN, et al. Randomized phase III trial of docetaxel versus vinorelbine or ifosfamide in patients with advanced non-small-cell lung cancer previously treated with platinum-containing chemotherapy regimens. The TAX 320 Non-Small Cell Lung Cancer Study Group. J Clin Oncol. 2000;18(12):2354-2362.

9. Shepherd FA, Dancey J, Ramlau R, et al. Prospective randomized trial of docetaxel versus best supportive care in patients with non-small-cell lung cancer previously treated with platinum-based chemotherapy. J Clin Oncol. 2000;18(10):2095-2103.

10. Tfayli A, Rafei H, Mina A, et al. Prevalence of EGFR and ALK mutations in lung adenocarcinomas in the levant area - a prospective analysis. Asian Pac J Cancer Prev. 2017;18(1):107-114.

11. Lin JJ, Cardarella S, Lydon CA, et al. Five-year survival in EGFRmutant metastatic lung adenocarcinoma treated with EGFR-TKIs. J Thorac Oncol. 2016;11(4):556-565.
12. Kris MG, Natale RB, Herbst RS, et al. Efficacy of gefitinib, an inhibitor of the epidermal growth factor receptor tyrosine kinase, in symptomatic patients with non-small cell lung cancer: a randomized trial. JAMA. 2003;290(16):2149-2158.

13. Brahmer JR, Tykodi SS, Chow LQ, et al. Safety and activity of antiPD-L1 antibody in patients with advanced cancer. $N$ Engl $J$ Med. 2012;366(26):2455-2465.

14. Dai S, Jia R, Zhang X, Fang Q, Huang L. The PD-1/PD-Ls pathway and autoimmune diseases. Cell Immunol. 2014;290(1):72-79.

15. Pardoll DM. The blockade of immune checkpoints in cancer immunotherapy. Nat Rev Cancer. 2012;12(4):252-264.

16. Garon EB, Rizvi NA, Hui R, et al. Pembrolizumab for the treatment of non-small-cell lung cancer. $N$ Engl J Med. 2015;372(21): 2018-2028.

17. Gettinger SN, Horn L, Gandhi L, et al. Overall survival and long-term safety of nivolumab (anti-programmed death 1 antibody, BMS-936558, ONO-4538) in patients with previously treated advanced non-small-cell lung cancer. J Clin Oncol. 2015;33(18):2004-2012.

18. Silverman LR, Greenberg P, Raza A, et al. Clinical activity and safety of the dual pathway inhibitor rigosertib for higher risk myelodysplastic syndromes following DNA methyltransferase inhibitor therapy. Hematol Oncol. 2015;33(2):57-66.

19. Tharyan P, Clarke M, Green S. How the Cochrane collaboration is responding to the Asian tsunami. PLoS Med. 2005;2(6):e169.

20. Begg CB, Mazumdar M. Operating characteristics of a rank correlation test for publication bias. Biometrics. 1994;50(4):1088-1101.

21. Stuck AE, Rubenstein LZ, Wieland D. Bias in meta-analysis detected by a simple, graphical test. Asymmetry detected in funnel plot was probably due to true heterogeneity. BMJ. 1998;316(7129):470-461.

22. Eisenhauer EA, Therasse P, Bogaerts J, et al. New response evaluation criteria in solid tumours: revised RECIST guideline (version 1.1). Eur J Cancer. 2009;45(2):228-247.

23. Herbst RS, Baas P, Kim DW, et al. Pembrolizumab versus docetaxel for previously treated, PD-L1-positive, advanced non-small-cell lung cancer (KEYNOTE-010): a randomised controlled trial. Lancet. 2016;387(10027):1540-1550.

24. Borghaei H, Paz-Ares L, Horn L, et al. Nivolumab versus docetaxel in advanced nonsquamous non-small-cell lung cancer. $N$ Engl $\mathrm{J}$ Med. 2015;373(17):1627-1639.

25. Brahmer J, Reckamp KL, Baas P, et al. Nivolumab versus docetaxel in advanced squamous-cell non-small-cell lung cancer. $N$ Engl J Med. 2015;373(2):123-135.

26. Rittmeyer A, Barlesi F, Waterkamp D, et al. Atezolizumab versus docetaxel in patients with previously treated non-small-cell lung cancer (OAK): a phase 3, open-label, multicentre randomised controlled trial. Lancet. 2017;389(10066):255-265.

27. Fehrenbacher L, Spira A, Ballinger M, et al. Atezolizumab versus docetaxel for patients with previously treated non-small-cell lung cancer (POPLAR): a multicentre, open-label, phase 2 randomised controlled trial. Lancet. 2016;387(10030):1837-1846.

28. Madore J, Vilain RE, Menzies AM, et al. PD-L1 expression in melanoma shows marked heterogeneity within and between patients: implications for anti-PD-1/PD-L1 clinical trials. Pigment Cell Melanoma Res. 2015;28(3):245-253.

29. Taube JM, Anders RA, Young GD, et al. Colocalization of inflammatory response with B7-h1 expression in human melanocytic lesions supports an adaptive resistance mechanism of immune escape. Sci Transl Med.2012; 4(127):127ra37.

30. Huang AC, Postow MA, Orlowski RJ, et al. T-cell invigoration to tumour burden ratio associated with anti-PD-1 response. Nature. 2017; 545(7652):60-65.

31. Azuma K, Ota K, Kawahara A, et al. Association of PD-L1 overexpression with activating EGFR mutations in surgically resected nonsmallcell lung cancer. Ann Oncol. 2014;25(10):1935-1940.

32. Calles A, Liao X, Sholl LM, et al. Expression of PD-1 and its ligands, PD-L1 and PD-L2, in smokers and never smokers with KRAS-mutant lung cancer. J Thorac Oncol. 2015;10(12):1726-1735. 
33. Dupage M, Cheung AF, Mazumdar C, et al. Endogenous T cell responses to antigens expressed in lung adenocarcinomas delay malignant tumor progression. Cancer Cell. 2011;19(1):72-85.
34. Wang C, Yu X, Wang W. A meta-analysis of efficacy and safety of antibodies targeting PD-1/PD-L1 in treatment of advanced nonsmall cell lung cancer. Medicine. 2016;95(52):e5539.

\section{Publish your work in this journal}

OncoTargets and Therapy is an international, peer-reviewed, open access journal focusing on the pathological basis of all cancers, potential targets for therapy and treatment protocols employed to improve the management of cancer patients. The journal also focuses on the impact of management programs and new therapeutic agents and protocols on

patient perspectives such as quality of life, adherence and satisfaction. The manuscript management system is completely online and includes a very quick and fair peer-review system, which is all easy to use. Visit http://www.dovepress.com/testimonials.php to read real quotes from published authors.

\footnotetext{
Submit your manuscript here: http://www.dovepress.com/oncotargets-and-therapy-journal
} 